\title{
A new predictor-corrector method for the numerical solution of fractional differential equations
}

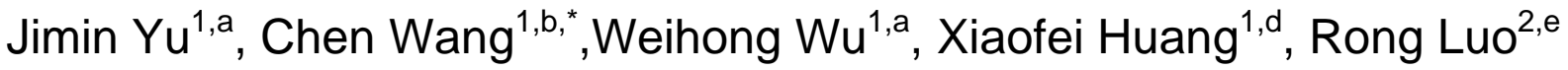 \\ ${ }^{1}$ Automation College, Chongqing University of Posts and Telecommunications, Chongqing 400065, \\ China \\ ${ }^{2}$ Advanced Manufacturing Engineering College, Chongqing University of Posts and \\ Telecommunications, Chongqing 400065, China \\ ayujm@cqupt.edu.cn, ${ }^{\text {b.*}}{ }^{*} 812303775 @ q q . c o m$,
}

Keywords: fractional differential equation, Caputo derivative, Adams method, predictor-corrector method, modified predictor-corrector method

\begin{abstract}
In this paper, first, a new predictor-corrector method( called the modified predictorcorrector method ) for the fractional differential equations is developed. And the new method can be formally viewed as a modification as the classical predictor-corrector method. Second, it is proved that the numerical accuracy of this new method is superior to that of the classical predictorcorrector method by two numerical examples. The comparison with the corresponding results demonstrates that the modified predictor-corrector method is more accurate than the classical predictor-corrector method when solving fractional differential equations numerically.
\end{abstract}

\section{Introduction}

In recent years, fractional calculus has got dramatic advances in both theory and application. For the no local property, the fractional calculus has been used to describe the basic nature of almost all sciences and engineering fields, such as fluid flow in porous materials, anomalous diffusion transport, acoustic wave propagation in viscoelastic materials, dynamics in self-similar structures, signal processing, financial theory, electric conductance of biological systems and so on[1].

However, the development process of numerical algorithm of fractional differential equations is slow at work. In 1986, Lubich[2] firstly promoted the BDF into the numerical calculation of the fractional differential equations and gained the form of the fractional BDF. And in 1993,Lubich and Ostermann[3] gained a high order approximation scheme for solving the fractional differential equations. And in 1998-2002 Diethiem[4-5]pointed an Adams-type predictor-corrector method for the numerical solution of fractional differential equations and the corresponding error analysis. In 2006, Odibat[6]presented an algorithm to numerically approximate the fractional integration and Caputo fractional differentiation. In 2011, Li, Chen and Ye[7] proposed some high-order numerical approximations for fractional integrals based on cubic Hermite interpolation and cubic spline interpolation. In 2013, Gao[8] proposed a method to approximate the Caputo fractional derivative by the quadratic interpolation.

The plan of the remainder is as follows. In Section2, a new Adams-type predictor-corrector method( called the modified predictor-corrector method ) for the fractional differential equations is developed. In Section3, one test examples are used to confirm the numerical accuracy of the new method. The computational results are compared with the corresponding ones with the classical predictor-corrector method. Finally, a brief conclusion and the further work have been listed.

\section{Derivation of the modified predictor-corrector method}

In this section, we will describe the classical predictor-corrector method and the process of the derivation of the modified predictor-corrector method in detail. Consider the fractional differential equation with initial conditions 
$\left\{\begin{array}{l}D_{*}^{\alpha} y(t)=f(t, y(t)) \\ y^{(k)}(t)=y_{0}^{(k)} \quad k=0,1, \ldots m-1\end{array}\right.$

Equation (2.1) is equivalent to the Volterra integral equation

$y(t)=\sum_{k=0}^{[\alpha]-1} y_{0}^{(k)} \frac{t^{k}}{k !}+\frac{1}{\Gamma(\alpha)} \int_{0}^{t}(t-\tau)^{\alpha-1} f(\tau, y(\tau)) d \tau$

Set $h=T / N, t_{n}=n h, n \geq 0$, then Eq.(2.2) can be written as

$$
\begin{gathered}
y_{h}\left(t_{n+1}\right)=\sum_{k=0}^{[\alpha]-1} y_{0}^{(k)} \frac{t_{n+1}^{k}}{k !}+\frac{h^{\alpha}}{\Gamma(\alpha+2)} f\left(t_{n+1}, y_{h}^{\mathrm{P}}\left(t_{n+1}\right)\right) \\
+\frac{h^{\alpha}}{\Gamma(\alpha+2)} \sum_{j=0}^{n} a_{j, \mathrm{n}+1} f\left(t_{j}, y_{h}\left(t_{j}\right)\right)
\end{gathered}
$$

where

$a_{j, n+1}= \begin{cases}n^{\alpha}-(\mathrm{n}-\alpha)(\mathrm{n}+1)^{\alpha} & \text { if } j=0 \\ (\mathrm{n}+2-\mathrm{j})^{\alpha}+(\mathrm{n}-\mathrm{j})^{\alpha+1}-2(\mathrm{n}-\mathrm{j}+1)^{\alpha+1}, & \text { if } 1 \leq j \leq n \\ 1 & \text { if } j=n+1\end{cases}$

$y_{h}^{\mathrm{P}}\left(t_{n+1}\right)=\sum_{k=0}^{[\alpha]-1} y_{0}^{(k)} \frac{t_{n+1}^{k}}{k !}+\frac{1}{\Gamma(\alpha)} \sum_{j=0}^{n} b_{j, n+1} f\left(t_{j}, y_{h}\left(t_{j}\right)\right)$,

$b_{j, n+1}=\frac{h^{\alpha}}{\alpha}\left((\mathrm{n}+1-\mathrm{j})^{\alpha}-(\mathrm{n}-\mathrm{j})^{\alpha}\right)$.

The error is $\max _{j=0,1, \ldots, N}\left|x\left(t_{j}\right)-x_{h}\left(t_{j}\right)\right|=O\left(h^{p}\right)$, where $p=\min (2,1+\alpha)$.

Then the representation of the modified predictor-corrector method is

$$
\begin{gathered}
y_{h}\left(t_{n+1}\right)=\sum_{k=0}^{[\alpha]-1} y_{0}^{(k)} \frac{t_{n+1}^{k}}{k !}+\frac{h^{\alpha}}{\Gamma(\alpha+2)} f\left(t_{n+1}, y_{h}^{\mathrm{MP}}\left(t_{n+1}\right)\right) \\
+\frac{h^{\alpha}}{\Gamma(\alpha+2)} \sum_{j=0}^{n} a_{j, \mathrm{n}+1} f\left(t_{j}, y_{h}\left(t_{j}\right)\right)
\end{gathered}
$$

the $y_{h}^{\mathrm{MP}}\left(t_{n+1}\right)$ is obtained by using $f\left(t_{n}\right)+f^{\prime}\left(t_{n}\right)\left(t-t_{n}\right)$ as the approximation of the integrand $f(t)$ on small interval $\left[\mathrm{t}_{n}, \mathrm{t}_{n+1}\right](n \geq 1)$. The representation of $y_{h}^{\mathrm{MP}}\left(t_{n+1}\right)$ is

$$
\begin{aligned}
y_{h}^{\mathrm{MP}}\left(t_{n+1}\right)= & \sum_{k=0}^{[\alpha]-1} y_{0}^{(k)} \frac{t_{n+1}^{k}}{k !}+\frac{1}{\Gamma(\alpha)} \sum_{j=0}^{n} b_{\mathrm{j}, \mathrm{n}+1} f\left(t_{j}, y_{h}\left(t_{j}\right)\right) \\
& +\frac{1}{\Gamma(\alpha)} \sum_{j=1}^{n} c_{j, n+1}\left(f\left(t_{j}, y_{h}\left(t_{j}\right)\right)-f\left(t_{j-1}, y_{h}\left(t_{j-1}\right)\right)\right)
\end{aligned}
$$

where $\quad c_{j, n+1}=\frac{h^{\alpha}}{\alpha}\left(\frac{1}{\alpha+1}(\mathrm{n}+1-\mathrm{j})^{\alpha+1}+\frac{1}{\alpha+1}(\mathrm{n}-\mathrm{j})^{\alpha+1}-(\mathrm{n}-\mathrm{j})^{\alpha}\right)$

And $f\left(t_{n}\right)$ and $f\left(t_{n}\right)+f^{\prime}\left(t_{n}\right)\left(t-t_{n}\right)$ are respectively corresponding first order and second order in Taylor series. According to Taylor's theorem, the higher approximation of $f(t)$ will reach higher accuracy. Then we can make a conclusion that the modified predicted value $y_{h}^{\mathrm{MP}}\left(t_{n+1}\right)$ in (2.7) is with higher accuracy than the predicted value in (2.3). 


\section{Numerical examples}

In this section, the validity and numerical accuracy of the modified predictor-corrector method (2.7) are demonstrated by one test examples. Meanwhile, the corresponding computational results with the classical predictor-corrector method (2.5)are given for contrast.

Let $h=T_{0} / N$ and denote that $y\left(t_{n}\right)$ indicates the exact solution at $t=t_{n}, y_{h}^{1}\left(t_{n}\right)$ and $y_{h}^{2}\left(t_{n}\right)$ respectively indicate the numerical solution got by the classical predictor-corrector method and the modified predictor-corrector method and $y\left(t_{n}\right)$ is the exact solution., and $E_{1}^{N}(h)=\left|y\left(t_{N}\right)-y_{h}^{1}\left(t_{N}\right)\right|, \quad E_{2}^{N}(\mathrm{~h})=\left|y\left(t_{N}\right)-y_{h}^{2}\left(t_{N}\right)\right|$.

\section{Example 3.1}

$$
D_{*}^{\alpha} y(t)=\frac{40320}{\Gamma(9-\alpha)} t^{8-\alpha}-3 \frac{\Gamma(5+\alpha / 2)}{\Gamma(5-\alpha / 2)} t^{4-\alpha / 2}+\frac{9}{4} \Gamma(\alpha+1)+\left(\frac{3}{2} t^{\alpha / 2}-t^{4}\right)^{3}-[y(t)]^{3 / 2}
$$

where $0<\alpha<2$ and the initial conditions are $y(0)=0, y^{\prime}(0)=0$.The exact solution of this initial value problem is $y(t)=t^{8}-3 t^{4+\alpha / 2}+\frac{9}{4} t^{\alpha}$.

Taking different temporal step sizes, $h=1 / 100,1 / 400,1 / 1600$, we compute the example by the classical predictor-corrector method and the modified predictor-corrector method, respectively. Table 1 lists the computational errors at $t_{N}=T_{0}=0.6$ with different parameters $\alpha=0.9,0.75,0.5$. And the absolute errors curves and solution curves with $\alpha=0.75, N=600, h=1 / 1000$ is presented in Fig.1. From the results presented in Table 1 and Fig.1, we find that the computational errors by the modified predictor-corrector method (2.7) are obviously much smaller than that by the classical predictor-corrector method (2.3).
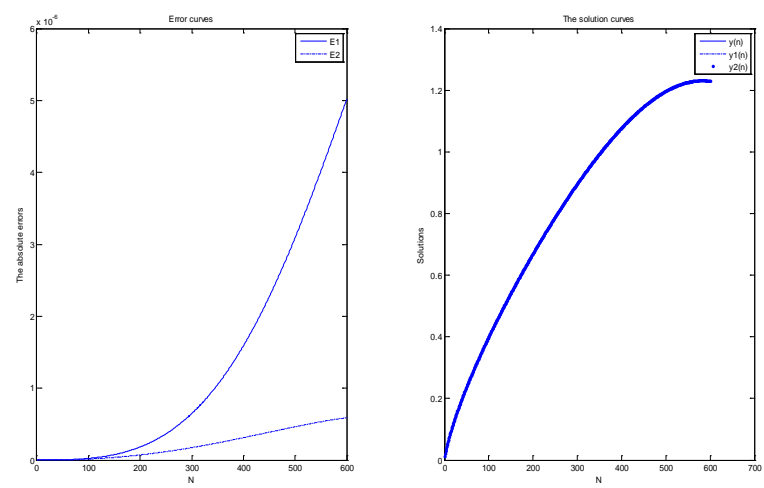

Fig. 1.The absolute errors and solution curves with $\alpha=0.75, N=600, h=1 / 1000$.

Table 1(example 3.1) Computational errors with different temporal step sizes.

\begin{tabular}{cccc}
\hline$\alpha$ & $h$ & $E_{1}^{N}(h)$ & $E_{2}^{N}(h)$ \\
\hline 0.9 & $1 / 100$ & $2.5731 \mathrm{e}-004$ & $6.52632 \mathrm{e}-005$ \\
& $1 / 400$ & $1.7493 \mathrm{e}-005$ & $3.72349 \mathrm{e}-006$ \\
& $1 / 1600$ & $1.21059 \mathrm{e}-006$ & $2.22915 \mathrm{e}-007$ \\
& & & \\
& $1 / 100$ & $3.50281 \mathrm{e}-004$ & $9.25708 \mathrm{e}-00$ \\
0.75 & $1 / 400$ & $2.9842 \mathrm{e}-005$ & $1.68487 \mathrm{e}-008$ \\
& $1 / 1600$ & $2.6067 \mathrm{e}-006$ & $2.80132 \mathrm{e}-009$ \\
& & & \\
0.5 & $1 / 100$ & $1.26753 \mathrm{e}-003$ & $1.19484 \mathrm{e}-006$ \\
& $1 / 400$ & $1.46559 \mathrm{e}-004$ & $1.02271 \mathrm{e}-006$ \\
& $1 / 1600$ & $177025 \mathrm{e}-005$ & $134997 \mathrm{e}-007$ \\
\hline
\end{tabular}




\section{Conclusion}

In this paper, a modified predictor corrector method for the fractional differential equations is developed. As what we have seen, the computational results of the modified predictor corrector method are superior to that of the classical predictor corrector method. And the modified predictor corrector method can also be used for numerically solving many other fractional differential equations with the Caputo derivatives. However, in this paper, there is only modification in the prediction part, which limiting the accuracy of the modified predictor corrector method. And in the future, efforts will be paid on the modification in the correction part, such as applying higher Lagrange interpolation instead of the linear interpolation to improve the accuracy.

\section{Reference}

[1] G.A. Anastassiou, Advances on fractional inequalities, Springer, 2011.

[2] C. Lubich, Discretized fractional calculus, SIAM J. Math. Anal. 17(1986)704-719.

[3] C. Lubich, A. Ostermann, Runge-Kutta methods for parabolic equations and convolution quadrature, Math. Comput. 60(1993)105-131.

[4] K. Diethelm, A. D. Freed, The FracPECE subroutine for the numerical solution of differential equations of fractional order, Forschung und wissenschaftliches Rechnen, 52 (1998): 57-71.

[5] K. Diethelm, N. J. Ford, A. D. Freed, A predictor-corrector approach for the numerical solution of fractional differential equations, Nonlinear Dynamics, 29 (2002) 3-22.

[6] Z. Odibat, Approximations of fractional integrals and Caputo fractional derivatives, Appl. Math. Comput. 178 (2006) 527-533.

[7] C. Li, A. Chen, J. Ye, Numerical approaches to fractional calculus and fractional ordinary differential equation, J. Comput. Phys. 230 (2011) 3352-3368.

[8] G. H. Gao, Z. Z. Sun, H. W. Zhang, A new fractional numerical differentiation formula to approximate the Caputo fractional derivative and its applications, J. Comput. Phys. 259(2014)33-50. 